\title{
Potentiale additiv gefertigter Gitterstrukturen - ein Review
}

\author{
Fiona Schulte ${ }^{1 *}$, Sebastian Weber ${ }^{2}$, Jannik Reichwein ${ }^{1}$, Kristin Paetzold ${ }^{2}$, \\ Eckhard Kirchner ${ }^{1}$ \\ 1 Institute for Product Development and Machine Elements, TU Darmstadt \\ 2 Institute for Technical Product Development, University of the Bundeswehr Munich \\ * Korrespondierender Autor: \\ Fiona Schulte \\ TU Darmstadt \\ Institute for Product Development and Machine Elements \\ Otto-Berndt-Straße 2 \\ 64287 Darmstadt \\ Telefon: +4961511621208 \\ Mail: schulte@pmd.tu-darmstadt.de
}

\begin{abstract}
Additive manufacturing (AM) offers a variety of potentials, especially the fabrication of lattice and cellular structures. The properties of lattices and cells can be influenced on purpose by designing their defined structure. To utilise the potentials in applications, it is important to identify them and point out possible applications with successful examples, which allow transferring approaches for the integration of lattice structures in components to new applications. Therefore, this contribution provides a collection of potentials of additively manufactured lattice structures from the state of research in a systematic review and examines their transferability to different applications.
\end{abstract}

\section{Keywords}

additive manufacturing, design for additive manufacturing, lattice structures, cell structures, potentials 


\section{Motivation}

Die additive Fertigung (AM) bietet eine Vielzahl von Potentialen. Insbesondere ermöglicht AM die Fertigung filigraner, komplexer Strukturen, u. a. Zell- und Gitterstrukturen, die im Folgenden unter dem Begriff Gitterstrukturen zusammengefasst werden. Durch die definierte Struktur der Gitter-Elementarzellen können Gitter gezielt in ihren Eigenschafen beeinflusst und somit optimiert werden. Diese Eigenschaften sind über die Topologie der Elementarzellen, ihre Dimension und deren Größe beeinflussbar [1].

Zur Nutzung der Potentiale von Gitterstrukturen in der Anwendung, ist es wichtig, die Potentiale zu identifizieren und Vorbilder erfolgreicher Nutzung von Gitterstrukturen aufzuzeigen, von denen Ansätze zur Integration der Gitterstrukturen in Bauteilen abgeleitet werden können. Daher werden in diesem Beitrag die Potentiale additiv gefertigter Gitterstrukturen aus dem Stand der Forschung systematisch analysiert und deren Anwendungen zusammengetragen. Aus den zusammengetragenen Beispielen können Ansätze in verschiedene Anwendungen übertragen werden. Wie etwa die Verwendung von Gittertypen, die für eine Anwendung bereits erfolgreich eingesetzt wurden, in eine andere Anwendung mit ähnlichen Anforderungen zu übernehmen.

\section{Forschungsproblem und Forschungsziel}

Additiv gefertigte Gitterstrukturen werden in der aktuellen Forschung im Hinblick auf vielseitige Aspekte untersucht. Dabei werden unterschiedliche Topologien von Elementarzellen hinsichtlich ihrer Eigenschaften, z. B. Steifigkeit oder Energieabsorption experimentell, numerisch oder in Use Cases untersucht. In den Veröffentlichungen werden stets ausgewählte Gitterstrukturen bezüglich ihrer Eignung für ausgewählte Zwecke betrachtet. Bislang fehlt jedoch ein Überblick über bereits genutzte Potentiale von Gitterstrukturen und Anwendungen, für die verschiedene Arten von Gitterstrukturen sich besonders eignen. Daher werden in diesem Beitrag die Potentiale additiv gefertigter Gitterstrukturen sowie Anwendungsbeispiele vorgestellt, um folgende Forschungsfrage zu adressieren: Welche Potentiale bieten additiv gefertigte Gitterstrukturen und für welche Anwendungen sind sie besonders geeignet?

\section{Methodisches Vorgehen}

Um eine nachvollziehbare Analyse der Veröffentlichungen im Bereich der Gitter- und Zellstrukturen zu präsentieren, wurde der Ansatz eines systematischen Reviews für die Literaturanalyse gewählt. Vom Regelfall abweichend werden die Ergebnisse der Analyse in Abschnitt 4 jedoch als Fließtext anstatt in einer tabellarischen Darstellung beschrieben.

\subsection{Vorgehen zur Literaturanalyse}

Für das systematische Review wurde das von KITCHENHAM et al. vorgestellte Vorgehen adaptiert und angewendet [2]. Dieses Vorgehen umfasst die fünf Schritte (1) Formulierung einer Forschungsfrage, (2) Literaturrecherche, (3) Bewertung der Validität der Ergebnisse, (4) erneute Bewertung unter Betrachtung der Ingenieursexpertise und (5) Auswertung der Effektivität in der Ausführung der Schritte (1) - (4). Die Forschungsfrage des ersten Schritts wurde dabei bereits im vorhergehenden Abschnitt formuliert.

\subsection{Quellenauswahl}

Der zweite Schritt des Vorgehens, die Literaturrecherche erfordert die Wahl einer Literaturdatenbank und die Definition eines Suchterms. Hierzu wurde die Forschungsfrage nach dem PICO-Modell (engl.: Problem, Intervention, Control und Outcome) [3] in vier Bestandteile zerlegt. Dieses Modell stammt ursprünglich aus der Analyse medizinischer Fragestellungen und 
musste auf den Ingenieursbereich angepasst werden. So ergeben sich die Bereiche Thema, Verfahren, Spezifizierung und Ergebnis. Der vierte Bereich bildet das Ergebnis der Literaturstudie und ist somit kein Bestandteil der Forschungsfrage. Die genaue Zerlegung der Forschungsfrage in die genannten Bestandteile erfolgt in Abschnitt 3.2.1.

Als Literaturdatenbank wurde auf das von Elsevier bereitgestellte Scopus ${ }^{\circledR}$ zurückgegriffen. Es umfasst eine Vielzahl wissenschaftlicher Beiträge verschiedener Verlage und bietet auch die Möglichkeit die Suche gezielt einzugrenzen. An dieser Stelle unterscheidet sich die Datenbank von dem weit verbreiteten Google Scholar, dessen Ergebnisse sich aufgrund der Suche im Volltext nur schwierig eingrenzen lassen.

\subsubsection{Auswahlkriterien}

Bei der Suche in Scopus ${ }^{\circledR}$ wurde der Suchbereich auf den Titel und die Kurzfassung der Veröffentlichungen begrenzt und dabei die Übereinstimmung von mindestens einem Stichwort je Bereich der Forschungsfrage als Kriterium definiert. Aus der Verknüpfung der Stichwörter mittels logischer Operatoren wurde ein Suchterm für die Literaturdatenbank generiert. Dabei wurden alle Stichworte eines Bereichs mit einem logischen ODER und alle Bereiche mit einem logischen UND verknüpft. Die Stichwörter wurden ausschließlich in englischer Sprache definiert, da dies auch auf die meisten Veröffentlichungen zutrifft. Für den Bereich Thema wurden die Begriffe lattice structure, cell ${ }^{*}$ structure, gyroid und unit cell gewählt, wobei das Sternsymbol (*) ein Platzhalter für verschiedene Schreibweisen ist. Stichwörter des Bereichs Verfahren sind alle gängigen Begriffe zur additiven Fertigung mit pulverbettbasierten Schmelzverfahren für Metalle und deren Abkürzungen. Der letzte Bereich dient zur Spezifizierung der Suchabfrage auf die Fragestellung des Forschungsproblems. Hierzu wurden neben verschiedenen Schreibweisen für Vergleich (compar*), Verhalten (behav*) und Eigenschaften (propert*) die Begriffe design, implementation, application und usage gewählt.

\subsubsection{Ausschlusskriterien}

Die Jahresspanne wurde auf die letzten 10 Jahre eingegrenzt, was in etwa dem Zeitraum der sich am Markt befindlichen Maschinen zur metallischen additiven Fertigung nach aktuellem Stand entspricht. Zur weiteren Eingrenzung wurden Ausschlusskriterien definiert. Diese umfassten Begriffe zu den Verfahren Materialextrusion, Stereolithografie und Elektronenstrahlschmelzen, deren Abkürzungen und geschützten Verfahrensbezeichnungen, Werkstoffen, speziell Kunststoffen, der Skala der Mikrostruktur (microstructure, grain und molecul*) und Eigenschaften durch Wärmebehandlungen der Mikrostruktur (heat-treatment), die im Rahmen dieser Veröffentlichung nicht berücksichtigt werden. Hier ist zu beachten, dass jedes dieser Ausschlusskriterien direkt zur Ausgrenzung aus der Ergebnismenge führt. Im Umkehrschluss bedeutet dies, dass evtl. Veröffentlichungen ausgeschlossen wurden, die neben dem relevanten Thema weitere irrelevante Themen behandeln.

\section{Auswertung der Literaturanalyse}

Für die Literaturrecherche wurden nur direkte Ergebnisse und nicht auch deren Referenzen verwendet. Die somit 2009 ausgegebenen Veröffentlichungen der Literatursuche wurden durch Anwendung der Ausschlusskriterien auf 515 relevante Veröffentlichungen reduziert. Durch manuelle Durchsicht der Kurzfassungen der jeweiligen Beiträge konnte die Ergebnismenge weiter auf 241 verbleibende Veröffentlichungen reduziert werden. In diesem Schritt wurden die Beiträge zudem in verschiedene Themengebiete gruppiert. Da das Themengebiet der Medizintechnik dabei den größten Anteil ausmacht, sollten diese Veröffentlichungen gesondert betrachtet werden und wurden in diesem Review nicht betrachtet. Ein weiterer Grund für den Ausschluss medizinischer Anwendungen liegt darin, dass Zellstrukturen hier primär für 
eine verbesserte Integration in das Knochenwachstum verwendet werden. Dies wird häufig durch nicht-periodische, schwammartige Zellen erreicht, welche für andere Anwendungen weniger Vorteile bieten als periodische Zellstrukturen. Um Beiträge ohne wissenschaftliche Qualitätssicherung in Form von Peer-Reviews auszuschließen, wurden zudem Konferenzbeiträge für diese Literaturstudie nicht betrachtet. Daraus wurde die Anzahl relevanter Veröffentlichungen weiter auf 97 Beiträge reduziert. Diese Beiträge wurden im Volltext auf Anwendungen und Potentiale von Gitter- und Zellstrukturen analysiert. Anhand der Volltexte ergaben sich weitere 25 Veröffentlichungen, die für die Fragestellungen dieser Studie irrelevant waren. Somit umfasst das Review nach allen Ausschlusskriterien und Eingrenzungen 72 Beiträge.

\subsection{Einheitszellen und periodische Gitter}

Gitter- und Zellstrukturen setzen sich in der Regel aus einer periodischen Anordnung von Einheitszellen zusammen. Diese Einheitszellen können entweder als Gitterzellen oder als dreifach periodische Minimalflächen (engl.: Triple Periodic Minimal Surfaces, TPMS) auftreten. Für Gitterzellen wird häufig die Bezeichnung von Kristallgittern verwendet. Gängige Zellen sind hier kubisch-raumzentrierte (BCC), kubisch-flächenzentrierte (FCC), einfach kubische (SC) Zellen oder Raumfachwerke (OT). Neben den geläufigen Zelltypen wurden bei den untersuchten Veröffentlichungen auch kubisch-kantenzentrierte (ECC) und hexagonale Zellen verwendet. Als meist verwendete TPMS-Zellen wurden das Schoen Gyroid, das Schwarz P (primitive) und das Schwarz D (diamond) ermittelt. Neben Gitterzellen und TPMS werden auch individuell gestaltete Einheitszellen, sowie zweidimensionale Anordnungen, die als Wabenstrukturen bezeichnet werden, beschrieben. Eine genaue Verteilung der in den Veröffentlichungen verwendeten Einheitszellen ist in Abbildung 1 gezeigt.

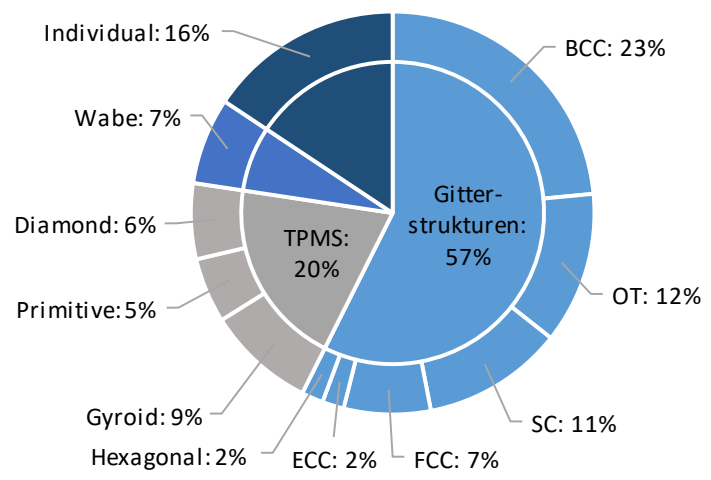

Abbildung 1: Verteilung der Einheitszellen bei den Gitter- und Zellstrukturen der untersuchten Veröffentlichungen

\subsection{Potentiale von Gitterstrukturen}

Im Nachfolgenden werden die Veröffentlichungen auf Potentiale der Gitter- und Zellstrukturen analysiert. Dabei wurden die Potentiale in sechs Bereiche untergliedert. Der größte dieser Bereiche ist Leichtbau, dem $29 \%$ der untersuchten Veröffentlichungen zugeteilt werden. Danach folgen Potentiale aus dem Bereich der Wärmeübertragung mit $18 \%$, sowie mit je $15 \%$ programmierbare Eigenschaften und Potentiale zur Rubrik Schwingungen. Neben der Energieabsorption mit 14\% Anteil wurden auch Potentiale zu optischen, chemischen und weiteren Anwendungsgebieten ermittelt, die in einem Abschnitt zusammengefasst wurden und $8 \%$ der Potentiale ausmachen.

\subsubsection{Leichtbau}

Gitterstrukturen sind aufgrund ihrer partiellen, zielgerichteten Materialverteilung besonders für Leichtbauweise geeignet. In den betrachteten Publikationen werden vielfach Anwendungen 
betrachtet, bei denen eine Optimierung der Funktion mit einer Steigerung des Leichtbauverhältnisses verbunden werden kann. Beispielsweise bei der Geometrieoptimierung [4] und Optimierung der Kühlung [5] eines Verbrennungsmotorzylinders und der Optimierung der Kühlung von Spritzgussformen [6]. Weitere Anwendung finden spezielle Gitter, etwa Kagome-Gitter, als aktorisch anwendbare Geometrien bei gleichzeitig geringer Masse [7] und für Knochenmaterial, bei dem sowohl die Masse als auch die mechanischen Eigenschaften mit Gitterstrukturen ähnlich denen menschlicher Knochen eingestellt werden können [8]. Häufig wird die Verbesserung von Energieabsorptions- bzw. Vibrationsdämpfungseigenschaften untersucht, etwa bei einem Fräswerkzeug, dessen Lebensdauer durch die reduzierte Vibration verlängert wird [9]. Die Reduktion von Vibrationen durch die Strukturgeometrie erlaubt in vielen Fällen eine Einsparung von Material und somit verbesserte Leichtbaueigenschaften. In diesem Bereich werden außerdem viele Untersuchungen durchgeführt, die nicht direkt auf eine Anwendung bezogen sind, aber unterschiedliche Gittertypen bzw. neu entwickelte Gitterstrukturen hinsichtlich ihrer Vibrationsdämpfung vergleichen [10-15].

Im Bereich der Leichtbaustrukturen, wie sie v. a. in der Luft- und Raumfahrt verwendet werden [16], wird die Optimierung der Leichtbaueigenschaften von Sandwichstrukturen durch die Verwendung von Gitterstrukturen als Kerne angestrebt. Dabei werden häufig Honigwaben [17] mit Gitterstrukturen, u. a. Diamant-Gittern [18], verglichen [19]. Auf diese Weise wurden Verbesserungen der Biege- und Drucksteifigkeit sowie der Energieabsorption erreicht [20].

Auch außerhalb der Mechanik bieten Gitterstrukturen Möglichkeiten, Funktionen zu optimieren und gleichzeitig Gewicht einzusparen, z. B. die Nutzung elektrischer Leitfähigkeit, deren Beschreibung von LAMBIN et al. untersucht wurde [21].

Um die Leichtbaupotentiale von Gitterstrukturen auszuschöpfen, wurden unterschiedliche Methoden entwickelt. Diese decken den Entwicklungsprozess in den frühen Phasen, sowie die Optimierung von Gitterstrukturen für den Einsatz im Bauteil [22, 23], für Leichtbau [24] sowie von den Zellen selbst [25, 26] ab. Für die Umsetzung von Leichtbau mithilfe von Gitterstrukturen wird überdies deren Herstellung und insbesondere Dimensionstreue untersucht [27]. Eine weitere Anwendung von Gitterstrukturen, die indirekt funktionsrelevant für ein additiv gefertigtes Bauteil ist, sind Stützkonstruktionen. Diese werden auf eine geringe Masse optimiert, um möglichst wenig Material entsorgen zu müssen [28, 29].

\subsubsection{Schwingungen}

Wie bereits im Abschnitt Leichtbau angesprochen wird häufig die Dämpfung von Vibrationen durch die Verwendung von Gitterstrukturen untersucht. Dabei steht meist im Fokus möglichst gute Vibrationsdämpfungseigenschaften zu erzeugen, etwa durch speziell entwickelte Zellen [12, 30]. Des Weiteren werden Zellen dahingehend ausgewählt und ausgelegt, dass sie möglichst große Bandbreiten an Schwingungen dämpfen, u. a. durch resonante Phononische Kristalle (PnC) [31] und Topologieoptimierung von Gitterstrukturen [32]. Eine weitere Möglichkeit, die Schwingungsabsorptionseigenschaften zu verbessern, sind eine gezielte Auslegung auf die Absorption von Eigenfrequenzen im System [33] und das Verschieben der StrukturEigenfrequenz in einen möglichst hohen Frequenzbereich [34, 35].

Neben periodischen Schwingungen sind Gitterstrukturen auch im Bereich der Stoßabsorption anwendbar. Dabei greifen die speziellen Versagensmechanismen bestimmter Gitterstrukturen wie TPMS-Gitterstrukturen [36] oder speziell entwickelter Strukturen, z. B. DoppelhelixGitterstrukturen [37]. Eine weitere Optimierung der Stoßabsorptionseigenschaften kann durch die Anpassung der SLM-Prozessparameter realisiert werden [38].

Die verbesserten Schwingungsabsorptionseigenschaften bringen bei Anwendungen Vorteile, wie eine Erhöhung der Lebensdauer etwa im Werkzeugbereich [9] oder Geräuschminderung z. B. durch Reduktion von Zahnradvibrationen in Getrieben [39]. 


\subsubsection{Programmierbare Eigenschaften}

Ein weiteres Potential der Gitterstrukturen ist die Möglichkeit das Verhalten von Bauteilen über die Eigenschaften der Elementarzellen zu beeinflussen. Dies ist möglich für mechanische Eigenschaften, wie der Steifigkeit und Festigkeit von Bauteilen, die durch eine Variation der Dichte, lokal im Bauteil angepasst werden kann [40, 41]. Ein weiterer Ansatz variiert die Einheitszelle im Bauteil entsprechend dem Belastungszustand im Bauteil [42]. Dies ermöglicht auch die gezielte Nutzung von Anisotropie in der Bauteilgestaltung. [43]

Auch die Querkontraktionszahl kann durch die gezielte Wahl der Elementarzelle beeinflusst werden, bis hin zu auxetischen Materialeigenschaften, also einer negativen Querkontraktionszahl [44, 45]. Die Querkontraktionszahl muss dabei nicht homogen über das Bauteil verteilt sein, sondern kann einem definierten Verlauf folgen. Dies kann beispielsweise in Anwendungen der Robotik zum Greifen von Gegenständen eingesetzt werden [46]. Ein weiterer Anwendungsfall für die gezielte Einstellung mechanischer Eigenschaften ist die Gestaltung einer peristaltischen Pumpe. [47] Die Beeinflussung der Eigenfrequenz von Bauteilen führt häufig zu sehr steifen und damit schweren Konstruktionen. Die Eigenfrequenz kann jedoch auch über die Elementarzelle beeinflusst werden. [27, 34]

Für die Auswahl und Gestaltung von Elementarzellen mit diesen spezifischen Eigenschaften gibt es beispielsweise Ansätze zur Verschachtelung von Einheitszellen, um deren Eigenschaften zu kombinieren [48, 49]. Darüber hinaus gibt es Ansätze, die sich mit der automatisierten Generierung von Einheitszellen auf Basis neuronaler Netze beschäftigen, um gezielt Materialeigenschaften zu erreichen [50]. Ein weiterer interessanter Anwendungsfall von programmierbaren Materialeigenschaften, sind Gitter, deren Eigenschaften durch ein Fernfeld beeinflusst werden können und sich somit ferngesteuert verformen lassen. [51]

\subsubsection{Energieabsorption}

Gitterstrukturen zeichnen sich darüber hinaus durch eine hohe spezifische Energieaufnahme aus [52]. Außerdem ermöglichen sie im Vergleich mit Vollmaterial eine größere Verformung des Bauteils bevor es zu plastischen Verformungen kommt [53]. Ein Vergleich von Gitterstrukturen mit konventionellen Waben in Sandwichbauteilen zeigt, dass beide Strukturen eine vergleichbare spezifische Energieabsorption aufweisen [54]. Dabei zeigt sich außerdem, dass die Energieaufnahme der Gitterstruktur abhängig von den Prozessparametern ist [38].

Für die Verbesserung der Energieabsorption sollten stabförmige Gitter plattenförmigen vorgezogen werden $[55,56]$. Stab-Oktaeder weisen bessere Beständigkeit gegen Explosionen auf als kubisch-raumzentrierte Gitter [57] und eine höhere Energieaufnahme als kubisch-flächenzentrierte Gitter [10]. Bei einem Vergleich der TPMS-Gitter sind Primitive- den GyroidStrukturen vorzuziehen [36] Eine andere Untersuchung zeigt, dass FBCCZ-Strukturen den höchsten Einschlagwiderstand aufweisen [58]. Insgesamt können die Ergebnisse der einzelnen Elementarzellen in den ausgewerteten Beiträgen nur schwer miteinander verglichen werden, da unterschiedliche Materialien, Prozessparameter und Testverfahren angewandt wurden.

Die Energieaufnahme kann jedoch durch einen an den Belastungszustand angepassten Strebendurchmesser im Vergleich zu BCC-Gittern mit homogenem Strebendurchmesser verbessert werden [25]. Dies kann beispielsweise durch die Topologieoptimierung der Einheitszelle erreicht werden [11]. Die Energieabsorption kann auch durch die Verwendung von Deckschichten in Form einer Sandwichstruktur verbessert werden [57].

\subsubsection{Wärmeübertragung}

Ein Hauptanwendungsgebiet von Zell- und Gitterstrukturen im Bereich der Wärmeübertragung sind Kühlkörper. Das Potential der Gitterstrukturen kann weiter in aktive und passive Kühlung untergliedert werden. Ho et al. ermitteln geometrische Parameter von Kühlkanälen 
mit rhombisch-oktaedrischen Zellen für eine wassergekühlte Kühlplatte. Neben der durch Wasser gekühlten Anwendung wurden mit dem gleichen Zelltyp auch experimentelle Versuche im Windkanal durchgeführt und die Wärmeübertragung mit herkömmlichen Kühlrippen verglichen [59]. Erzwungene Konvektion ist ebenfalls Thema der Veröffentlichung von AL-KETAN et al. [60]. Sie vergleichen Strömungs- und Wärmetransporteigenschaften von TPMS-Zellen (Gyroid und Diamant) mithilfe von Strömungssimulationen. Neben der Simulation wenden TAKEZAWA et al. auch Optimierungsalgorithmen an, um die Gitterverteilung zu verbessern [61]. Ziel dabei ist eine thermische und mechanische Optimierung, sowie die Strömungsoptimierung für Kühlkörper aus Gitterstrukturen mit kubisch-flächenzentrierten Einheitszellen. Die passive Kühlung durch freie Konvektion hingegen wird von VAISSIER et al. behandelt [62]. Am Beispiel einer Ölwanne wurden kubisch-flächenzentrierte, -raumzentrierte, einfach kubische und Oktaeder Einheitszellen auf ihre Wärmeleitungs- und Konvektionseigenschaften untersucht. Zusätzlich wurde ein Framework zur parametrischen Konstruktion solcher Kühlstrukturen präsentiert. CHANTZIS et al. befassen sich ebenfalls mit dem Potential der verbesserten Kühlung [63]. In deren Veröffentlichung werden Wabenstrukturen in das Design von Stanzwerkzeugen integriert, um eine verbesserte Kühlung zu gewährleisten.

Das Potential des Wärmetransports wird unter anderem von KAUR UND SINGH für einfach kubische und oktaedrische Einheitszellen beschreiben [64]. Beispielhafte Anwendungen sind Katalysatoren [65] oder Kolben [5] im Fahrzeugbau. Wärmeabtransport durch ein verbessertes Siedeverhalten mithilfe von Gitterstrukturen ist Thema der Veröffentlichungen von WoNG und LEONG $[66,67]$. Sie untersuchen verbesserte Wärmeübergangseigenschaften durch eine erhöhte Oberfläche und Gitterdichte zur Bläschenbildung, sowie durch Kapillarwirkung am Beispiel von Elektronikbauteilen. Die gezielte Anpassung von Geometrieparametern der verwendeten oktaedrischen Einheitszelle ermöglicht zudem eine präzise Steuerung des Siedeverhaltens. Eine solche Beeinflussung der Wärmeableitung ist auch ein Potential der Veröffentlichung von CATCHPOLE-SMITH et al. [68], in der eine gerichtete Wärmeableitung mit in ein Bauteil integrierten TPMS-Zellen untersucht wird. Als Ergebnis wird deutlich, dass das SchwarzPrimitiv eine bessere Wärmeleitung besitzt als Gyroid- und Diamant-Einheitszellen.

Ein weiteres Potential ist ein verbessertes thermo-mechanische Verhalten, das von WU UND TOVAR diskutiert wird [69]. In der Veröffentlichung wird eine multiskalare thermisch-mechanische Optimierung angewandt und basierend auf dem Dichteergebnis die Zellstruktur generiert. Somit wird auf Makroebene die Zelldichte und auf Mesoebene die Zellstruktur selbst optimiert. Als Beispiel wird eine Spritzgussform optimiert und somit eine Gewichtsersparnis von 30\% erreicht. Neben Leichtbau wird in WANG et al. die Hitzebeständigkeit thematisiert [16]. Es werden TPMS-Zellen und kubisch-raumzentrierte Zellen für Gitterdichten zwischen $40 \%$ und $80 \%$ auf ihre mechanischen und thermischen Eigenschaften untersucht.

\subsubsection{Potentiale weiterer Anwendungsgebiete}

Ein weiteres Anwendungsgebiet ist die Optik. Diamantförmige TPMS-Zellen wurden dabei als optische Verschlüsse eingesetzt. Daraus ergibt sich das Potential zur richtungsabhängigen Transmission von Licht [70]. Die Transmissionseigenschaften von Zellstrukturen werden auch in der Veröffentlichung von SREEDHAR et al. untersucht [71]. Die Eignung von verschiedenen TPMS-Zellen zur Filtrierung wird darin näher betrachtet. Auch eine erhöhte Korrosionsbeständigkeit ist ein Potential von Zellstrukturen. Dies wird von ŁosIEWICZ et al. ebenfalls für verschiedene TPMS-Zellen analysiert [72].

\section{Diskussion}

Neben der Zusammenstellung der verwendeten Zellentypen wurde der Anteil der Zellen, welche in den jeweiligen Veröffentlichungen untersucht wurden, über dem betrachteten Zeit- 
raum untersucht. Vereinfachend wurde dabei nicht nach den einzelnen Elementarzellen aufgeschlüsselt, sondern, ob es sich um Stab-, TPMS- oder individuell entworfene Elementarzellen handelt. Abbildung 2 zeigt das Ergebnis dieser Untersuchung. Das Diagramm zeigt zum einen, dass zu Beginn des betrachteten Zeitraums fast ausschließlich Stabgitter betrachtet wurden und die Variantenvielfalt der untersuchten Zellen mit der Zeit zunimmt. Zum anderen nimmt der Anteil an TPMS-Gittern stetig zu und macht im Jahr 2021 bereits den größten Anteil aus. Die Gesamtzahl an betrachteten Beiträgen ist dabei jedes Jahr gestiegen.

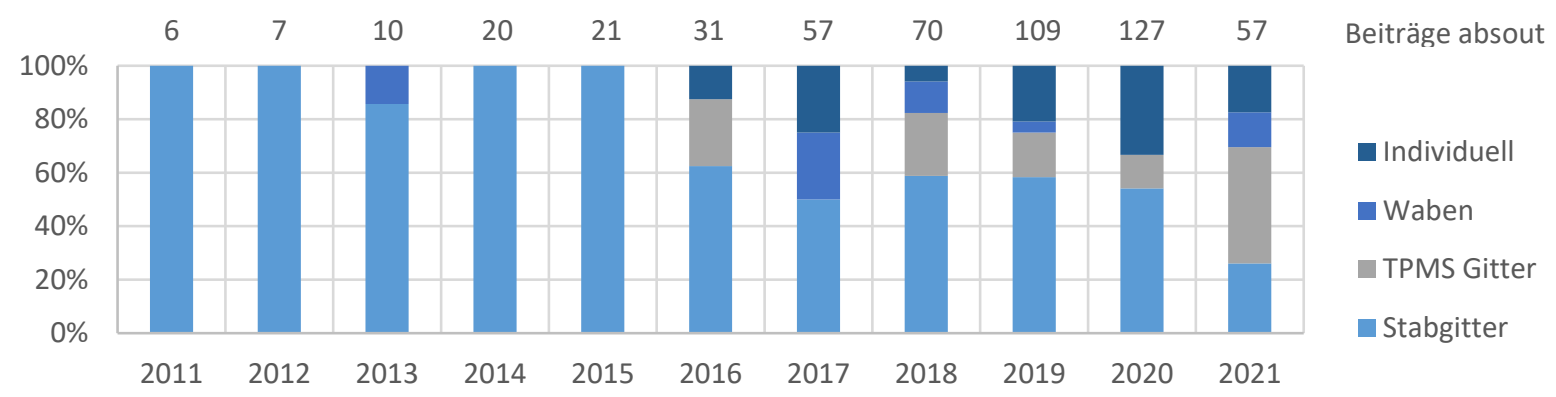

Abbildung 2: Anteil der untersuchten Elementarzellen über den betrachteten Zeitraum

Abbildung 3 zeigt die in den untersuchten Beiträgen genutzten Potentiale von Gitterstrukturen. Auch hier zeigt sich eine deutliche Ausweitung der Anwendungsfälle für Gitterstrukturen. Zu Beginn des untersuchten Zeitraums waren die Motive für den Einsatz von Gitterstrukturen vor allem die verbesserte Energieabsorption und das Leichtbaupotential. In den letzten Jahren werden Gitterstrukturen vor allem genutzt um spezifische, zeitlich veränderliche Materialeigenschaften zu erhalten.
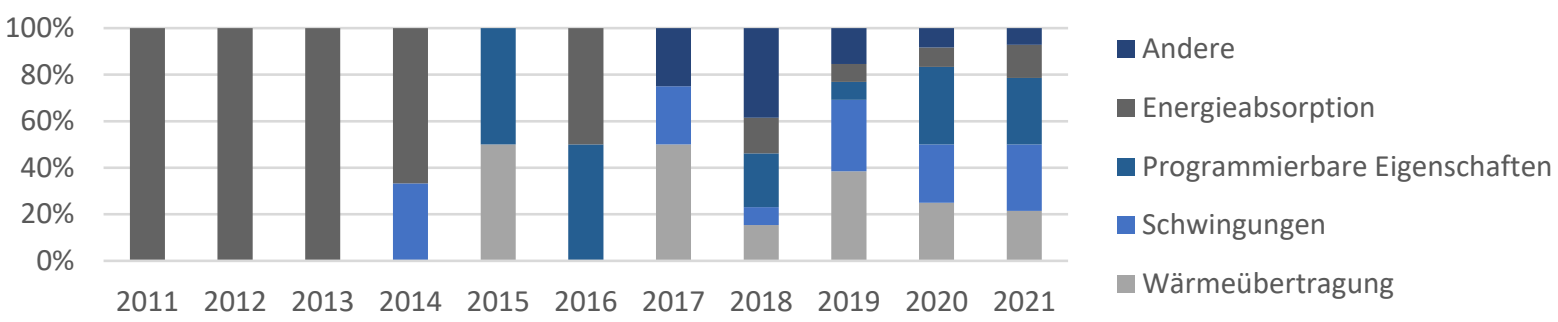

Abbildung 3: Anteil der genutzten Potentiale über den betrachteten Zeitraum

Abbildung 4 zeigt, welche Elementarzellen für welche Potentiale genutzt werden. Interessant ist vor allem, dass die herkömmlichen Stabgitter und TPMS-Gitter ungeeignet sind um programmierbare Eigenschaften zu erreichen. Hier müssen für den konkreten Anwendungsfall eigene Zellen entwickelt werden. Außerdem werden Stabgitter insbesondere für mechanische Anwendungen wie Leichtbau, Energieabsorption und Schwingungen genutzt. Im Gegensatz dazu werden TPMS insbesondere bei der Wärmeübertragung verwendet.

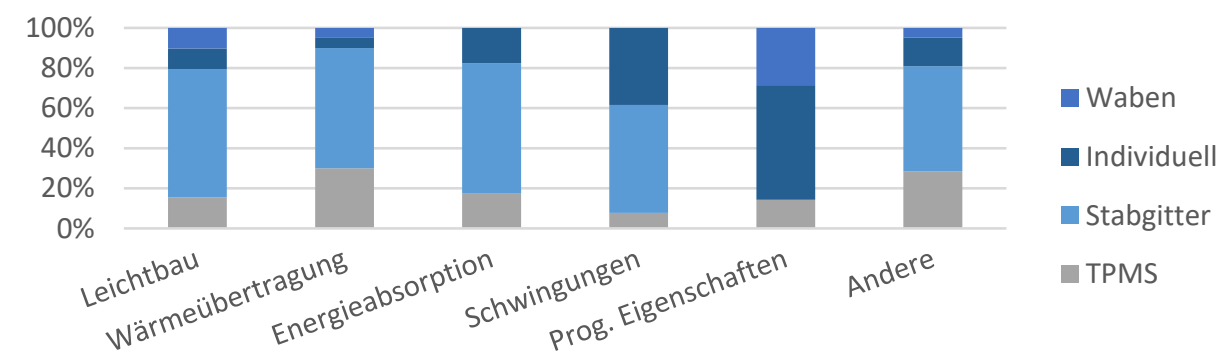

Abbildung 4: Anteil der Elementarzellen an den genutzten Potentialen. 


\section{Zusammenfassung und Ausblick}

Es wurden insgesamt 515 relevante Beiträge untersucht, von denen 72 Arbeiten Potentiale und Anwendungen von Gitterstrukturen thematisieren. Aus diesen 72 Arbeiten konnten insgesamt fünf häufig auftretende Potentiale herausgearbeitet werden, die durch Gitterstrukturen erreicht werden können. Diese sind Leichtbau, Wärmeübertragung, Energieabsorption, Schwingungen und programmierbare Eigenschaften.

Darüber hinaus wurde gezeigt, dass das Interesse an Gitterstrukturen in den letzten Jahren deutlich angestiegen ist. Dies führt auch zu breiter aufgestellten Forschungsvorhaben, die neue Elementarzellen und Potentiale thematisieren.

In Zukunft können weitere Elementarzellen entwickelt werden, die spezifische programmierbare Eigenschaften aufweisen, welche durch Vollmaterial nicht erreicht werden können. Außerdem sollten Anwendungen nicht nur eines der Potentiale nutzen, sondern wo möglich mehrere. Beispielsweise können Spritzgusswerkzeuge von der verbesserten Wärmeübertragung profitieren, und dadurch kürzere Taktzeiten ermöglichen. Zusätzlich kann das Werkzeug leichter gestaltet werden, was eine weitere Reduktion der Taktzeiten erlaubt. Die aufgezeigten Potentiale dienen daher als Inspiration und die entsprechenden Anwendungsfälle als konkrete Beispiele für die Umsetzung der Potentiale.

\section{Literaturverzeichnis}

[1] Berger, J. B. ; Wadley, H. N. G. ; McMeeking, R. M.: Mechanical metamaterials at the theoretical limit of isotropic elastic stiffness. In: Nature 543 (2017), Nr. 7646, S. 533-537

[2] Kitchenham, B. A. ; Dyba, T. ; Jorgensen, M.: Evidence-based software engineering. In: Proceedings. 26th International Conference on Software Engineering : IEEE Comput. Soc, 2004, S. 273-281

[3] Richardson, W. S. ; Wilson, M. C. ; Nishikawa, J. ; Hayward, R. S.: The well-built clinical question: a key to evidence-based decisions. In: ACP journal club 123 (1995), Nr. 3, A12-3

[4] Posser, Thiago ; Freitas de Oliveira, Branca: Design for additive manufacturing applied for mass reduction of a two-stroke engine cylinder for portable machine. In: International Journal on Interactive Design and Manufacturing (IJIDeM) 14 (2020), Nr. 2, S. 709-717

[5] Reyes Belmonte, Miguel Angel ; Copeland, Colin D. ; Hislop, Drummond ; Hopkins, George ; Schmieder, Adrian ; Bredda, Scott ; Akehurst, Sam: Improving Heat Transfer and Reducing Mass in a Gasoline Piston Using Additive Manufacturing. In: SAE Technical Paper Series : SAE International400 Commonwealth Drive, Warrendale, PA, United States, 2015 (SAE Technical Paper Series).

[6] Malca, C. ; Santos, C. ; Sena, M. ; Mateus, A.: Development of SLM cellular structures for injection molds manufacturing. In: Science and Technology of Materials 30 (2018), Nr. 1, S. 13-22

[7] Wei, Kai ; Yang, Qidong ; Ling, Bin ; Xie, Haiqiong ; Qu, Zhaoliang ; Fang, Daining: Mechanical responses of titanium 3D kagome lattice structure manufactured by selective laser melting. In: Extreme Mechanics Letters 23 (2018), Nr. 6366, S. 41-48

[8] Caiazzo, Fabrizia ; Alfieri, Vittorio ; Bujazha, Brahim David: Additive manufacturing of biomorphic scaffolds for bone tissue engineering. In: The International Journal of Advanced Manufacturing Technology 113 (2021), 9-10, S. 2909-2923

[9] Hanzl, Pavel ; Zetková, Ivana: Benefits of a New Approach to Designing Milling Cutter Using Metal Additive Manufacturing. In: Manufacturing Technology 19 (2019), Nr. 3, S. 385-390

[10] Osman, Mahmoud M. ; Shazly, Mostafa ; El-Danaf, Ehab A. ; Jamshidi, Parastoo ; Attallah, Moataz M.: Compressive behavior of stretched and composite microlattice metamaterial for energy absorption applications. In: Composites Part B: Engineering 184 (2020), Nr. 6190, S. 107715

[11] Xiao, Zefeng ; Yang, Yongqiang ; Xiao, Ran ; Bai, Yuchao ; Song, Changhui ; Di Wang: Evaluation of topology-optimized lattice structures manufactured via selective laser melting. In: Materials \& Design 143 (2018), S. $27-37$

[12] Doehring, Todd ; Nelson, William ; Harris, Thomas ; Freed, Alan: FE vibration analyses of novel conforming meta-structures and standard lattices for simple bricks and a topology-optimized aerodynamic bracket. In: Scientific reports 10 (2020), Nr. 1, S. 21484

[13] Dzogbewu, Thywill Cephas: Laser powder bed fusion of Ti6Al4V lattice structures and their applications. Journal of Metals, Materials and Minerals, 68-78 / Journal of Metals, Materials and Minerals, 30, 4, 68-78 (2020) 
[14] Zhang, L. ; Song, B. ; Fu, J. J. ; Wei, S. S. ; Yang, L. ; Yan, C. Z. ; Li, H. ; Gao, L. ; Shi, Y. S.: Topology-optimized lattice structures with simultaneously high stiffness and light weight fabricated by selective laser melting: Design, manufacturing and characterization. In: Journal of Manufacturing Processes 56 (2020), S. 1166-1177

[15] Boccini, Enrico ; Furferi, Rocco ; Governi, Lapo ; Meli, Enrico ; Ridolfi, Alessandro ; Rindi, Andrea ; Volpe, Yary: Toward the integration of lattice structure-based topology optimization and additive manufacturing for the design of turbomachinery components. In: Advances in Mechanical Engineering 11 (2019), Nr. 8, 168781401985978

[16] Wang, Xinglong ; Wang, Cheng ; Zhou, Xin ; Di Wang ; Zhang, Mingkang ; Gao, Yun ; Wang, Lei ; Zhang, Peiyu: Evaluating Lattice Mechanical Properties for Lightweight Heat-Resistant Load-Bearing Structure Design. In: Materials (Basel, Switzerland) 13 (2020), Nr. 21

[17] Zaharia, Sebastian Marian ; Lancea, Camil ; Chicos, Lucia Antoneta ; Pop, Mihai Alin ; Caputo, Giampaolo ; Serra, Emanuele: Mechanical Properties and Corrosion Behaviour of 316I Stainless Steel Honeycomb Cellular Cores Manufactured by Selective Laser Melting. In: Transactions of FAMENA 41 (2018), Nr. 4, S. 1124

[18] Yin, Xuan ; Meng, Wenjun ; Cheng, Jinzhao ; Wang, Hailong ; Zhao, Xiaoxia: A Kind of Filling Lattice Structure Based on DFAM for Mechanical Parts: The Diamond Lattice Structure. In: Advances in Materials Science and Engineering 2021 (2021), Nr. 10, S. 1-14

[19] Marschall, David ; Rippl, Herbert ; Ehrhart, Frank ; Schagerl, Martin: Boundary conformal design of laser sintered sandwich cores and simulation of graded lattice cells using a forward homogenization approach. In: Materials \& Design 190 (2020), Nr. 45, S. 108539

[20] Zhang, Jingwei ; Yanagimoto, Jun: Topology optimization of microlattice dome with enhanced stiffness and energy absorption for additive manufacturing. In: Composite Structures 255 (2021), S. 112889

[21] Lambin, Philippe ; Melnikov, Alexander ; Shuba, Mikhail: Electrokinetic Properties of 3D-Printed Conductive Lattice Structures. In: Applied Sciences 9 (2019), Nr. 3, S. 541

[22] Tang, Yunlong ; Kurtz, Aidan ; Zhao, Yaoyao Fiona: Bidirectional Evolutionary Structural Optimization (BESO) based design method for lattice structure to be fabricated by additive manufacturing. In: ComputerAided Design 69 (2015), Part A, S. 91-101

[23] Wang, Yiqiang ; Zhang, Lei ; Daynes, Stephen ; Zhang, Hongying ; Feih, Stefanie ; Wang, Michael Yu: Design of graded lattice structure with optimized mesostructures for additive manufacturing. In: Materials \& Design 142 (2018), Nr. 9, S. 114-123

[24] Nguyen, Jason ; Park, Sang-in ; Rosen, David: Heuristic optimization method for cellular structure design of light weight components. In: International Journal of Precision Engineering and Manufacturing 14 (2013), Nr. 6, S. 1071-1078

[25] Zhao, Miao ; Liu, Fei ; Fu, Guang ; Zhang, David Z. ; Zhang, Tao ; Zhou, Hailun: Improved Mechanical Properties and Energy Absorption of BCC Lattice Structures with Triply Periodic Minimal Surfaces Fabricated by SLM. In: Materials (Basel, Switzerland) 11 (2018), Nr. 12

[26] Messner, Mark C.: Optimal lattice-structured materials. In: Journal of the Mechanics and Physics of Solids 96 (2016), 2-3, S. 162-183

[27] Allevi, Gloria ; Capponi, Lorenzo ; Castellini, Paolo ; Chiariotti, Paolo ; Docchio, Franco ; Freni, Fabrizio ; Marsili, Roberto ; Martarelli, Milena ; Montanini, Roberto ; Pasinetti, Simone ; Quattrocchi, Antonino ; Rossetti, Robert ; Rossi, Gianluca ; Sansoni, Giovanna ; Tomasini, Enrico Primo: Investigating Additive Manufactured Lattice Structures: A Multi-Instrument Approach. In: IEEE Transactions on Instrumentation and Measurement 69 (2020), Nr. 5, S. 2459-2467

[28] Vaissier, Benjamin ; Pernot, Jean-Philippe ; Chougrani, Laurent ; Véron, Philippe: Genetic-algorithm based framework for lattice support structure optimization in additive manufacturing. In: Computer-Aided Design 110 (2019), Nr. 1, S. 11-23

[29] Xiao, Dong Ming ; Yang, Yong Qiang ; Su, Xu Bin ; Zhang, Man Hui ; Di Wang: Study on Cellular Structures as the Core Architectures of Parts Based on Selective Laser Melting. In: Advanced Materials Research 538541 (2012), S. 1904-1907

[30] Quinteros, Leonel ; Meruane, Viviana ; Cardoso, Eduardo Lenz: Phononic band gap optimization in trusslike cellular structures using smooth P-norm approximations. In: Structural and Multidisciplinary Optimization 64 (2021), Nr. 1, S. 113-124

[31] Meng, H. ; Bailey, N. ; Chen, Y. ; Wang, L. ; Ciampa, F. ; Fabro, A. ; Chronopoulos, D. ; Elmadih, W.: 3D rainbow phononic crystals for extended vibration attenuation bands. In: Scientific reports 10 (2020), Nr. 1, S. 18989

[32] Wormser, Maximilian ; Wein, Fabian ; Stingl, Michael ; Körner, Carolin: Design and Additive Manufacturing of 3D Phononic Band Gap Structures Based on Gradient Based Optimization. In: Materials (Basel, Switzerland) 10 (2017), Nr. 10 
[33] Zhao, Jiaqi ; Zhang, Ming ; Zhu, Yu ; Li, Xin ; Wang, Leijie ; Hu, Chuxiong: Concurrent optimization of additive manufacturing fabricated lattice structures for natural frequencies. In: International Journal of Mechanical Sciences 163 (2019), Nr. 10, S. 105153

[34] Andresen, Simone ; Bäger, Annette ; Hamm, Christian: Eigenfrequency maximisation by using irregular lattice structures. In: Journal of Sound and Vibration 465 (2020), S. 115027

[35] Cheng, Lin ; Liang, Xuan ; Belski, Eric ; Wang, Xue ; Sietins, Jennifer M. ; Ludwick, Steve ; To, Albert: Natural Frequency Optimization of Variable-Density Additive Manufactured Lattice Structure: Theory and Experimental Validation. In: Journal of Manufacturing Science and Engineering October 2018 (2018), Nr. 140

[36] Liang, Yingjing ; Zhou, Wei ; Liu, Yijie ; Li, Zhanshuo ; Yang, Yang ; Xi, Huifeng ; Wu, Zhigang: Energy Absorption and Deformation Behavior of 3D Printed Triply Periodic Minimal Surface Stainless Steel Cellular Structures under Compression. In: steel research international 92 (2021), Nr. 3, S. 2000411

[37] Hu, Jianxing ; Yu, T. X. ; Yin, Sha ; Xu, Jun: Low-speed impact mitigation of recoverable DNA-inspired double helical metamaterials. In: International Journal of Mechanical Sciences 161-162 (2019), Nr. 7, S. 105050

[38] Shen, Yiou ; Cantwell, Wesley ; Mines, Robert ; Li, Yan: Low-velocity impact performance of lattice structure core based sandwich panels. In: Journal of Composite Materials 48 (2014), Nr. 25, S. 3153-3167

[39] Ramadani, Riad; Pal, Snehashis ; Kegl, Marko ; Predan, Jožef ; Drstvenšek, Igor ; Pehan, Stanislav ; Belšak, Aleš: Topology optimization and additive manufacturing in producing lightweight and low vibration gear body. In: The International Journal of Advanced Manufacturing Technology 113 (2021), 11-12, S. 3389-3399

[40] Jin, Qing-Ye ; Yu, Jae-Hyun ; Ha, Kyung-Sik ; Lee, Wook-Jin ; Park, Sang-Hu: Multi-dimensional lattices design for ultrahigh specific strength metallic structure in additive manufacturing. In: Materials \& Design 201 (2021), S. 109479

[41] Wang, Yiqiang ; Zhang, Lei ; Daynes, Stephen ; Zhang, Hongying ; Feih, Stefanie ; Wang, Michael Yu: Design of graded lattice structure with optimized mesostructures for additive manufacturing. In: Materials \& Design 142 (2018), Nr. 9, S. 114-123

[42] Kang, Dongseok ; Park, Sanghu ; Son, Yong ; Yeon, Simo ; Kim, Sang Hoon ; Kim, llyong: Multi-lattice inner structures for high-strength and light-weight in metal selective laser melting process. In: Materials \& Design 175 (2019), Nr. 10, S. 107786

[43] Munford, Maxwell ; Hossain, Umar ; Ghouse, Shaaz ; Jeffers, Jonathan R.T.: Prediction of anisotropic mechanical properties for lattice structures. In: Additive Manufacturing 32 (2020), Nr. 4, S. 101041

[44] Guth, D. C. ; Luersen, M. A. ; Muñoz-Rojas, P. A.: Optimization of three-dimensional truss-like periodic materials considering isotropy constraints. In: Structural and Multidisciplinary Optimization 52 (2015), Nr. 5, S. 889-901

[45] Hedayati, Reza ; Güven, Aysun ; van der Zwaag, Sybrand: 3D gradient auxetic soft mechanical metamaterials fabricated by additive manufacturing. In: Applied Physics Letters 118 (2021), Nr. 14, S. 141904

[46] Han, Yafeng ; Lu, Wenfeng: Evolutionary design of nonuniform cellular structures with optimized Poisson's ratio distribution. In: Materials \& Design 141 (2018), Nr. 5, S. 384-394

[47] Weisheit, Linda ; Wenz, Franziska ; Lichti, Tobias ; Eckert, Medardus ; Baumann, Sascha ; Hübner, Christof ; Eberl, Christoph ; Andrä, Heiko: Domänenübergreifende Workflows zur effizienten Entwicklung Programmierbarer Materialien. In: Zeitschrift für wirtschaftlichen Fabrikbetrieb 115 (2020), 7-8, S. 470-475

[48] White, Benjamin C. ; Garland, Anthony ; Alberdi, Ryan ; Boyce, Brad L.: Interpenetrating lattices with enhanced mechanical functionality. In: Additive Manufacturing 38 (2021), Nr. 1, S. 101741

[49] Xu, Shanqing ; Shen, Jianhu ; Zhou, Shiwei ; Huang, Xiaodong ; Xie, Yi Min: Design of lattice structures with controlled anisotropy. In: Materials \& Design 93 (2016), S. 443-447

[50] Garland, Anthony P. ; White, Benjamin C. ; Jensen, Scott C. ; Boyce, Brad L.: Pragmatic generative optimization of novel structural lattice metamaterials with machine learning. In: Materials \& Design 203 (2021), Nr. 3, S. 109632

[51] Hedayati, R. ; Mirzaali, M. J. ; Vergani, L. ; Zadpoor, A. A.: Action-at-a-distance metamaterials: Distributed local actuation through far-field global forces. In: APL Materials 6 (2018), Nr. 3, S. 36101

[52] Harris, J. A. ; McShane, G. J.: Impact response of metallic stacked origami cellular materials. In: International Journal of Impact Engineering 147 (2021), S. 103730

[53] Davami, Keivan ; Mohsenizadeh, Mehrdad; Munther, Michael ; Palma, Tyler ; Beheshti, Ali ; Momeni, Kasra: Dynamic energy absorption characteristics of additively-manufactured shape-recovering lattice structures. In: Materials Research Express 6 (2019), Nr. 4, S. 45302

[54] Mines, R.A.W. ; Tsopanos, S. ; Shen, Y. ; Hasan, R. ; McKown, S. T.: Drop weight impact behaviour of sandwich panels with metallic micro lattice cores. In: International Journal of Impact Engineering 60 (2013), Nr. 3, S. 120-132 
[55] Winter, R. E. ; Cotton, M. ; Harris, E. J. ; Chapman, D. J. ; Eakins, D. ; McShane, G.: Plate-impact loading of cellular structures formed by selective laser melting. In: Schleyer, G.; Brebbia, C.A (Hrsg.): Structures Under Shock and Impact XII : WIT PressSouthampton, UK, 2012 (WIT Transactions on The Built Environment), S. $145-156$

[56] Winter, R. E. ; Cotton, M. ; Harris, E. J. ; Maw, J. R. ; Chapman, D. J. ; Eakins, D. E. ; McShane, G.: Plateimpact loading of cellular structures formed by selective laser melting. In: Modelling and Simulation in Materials Science and Engineering 22 (2014), Nr. 2, S. 25021

[57] Smith, M. ; Cantwell, W. J. ; Guan, Z. ; Tsopanos, S. ; Theobald, M. D. ; Nurick, G. N. ; Langdon, G. S.: The quasi-static and blast response of steel lattice structures. In: Journal of Sandwich Structures \& Materials 13 (2011), Nr. 4, S. 479-501

[58] Vrana, Radek; Koutny, Daniel ; Palousek, David: IMPACT RESISTANCE OF DIFFERENT TYPES OF LATTICE STRUCTURES MANUFACTURED BY SLM. In: MM Science Journal 2016 (2016), Nr. 06, S. 1579_ 1585

[59] Ho, J. Y. ; Leong, K. C. ; Wong, T. N.: Additively-manufactured metallic porous lattice heat exchangers for air-side heat transfer enhancement. In: International Journal of Heat and Mass Transfer 150 (2020), S. 119262

[60] Oraib Al-Ketan, Mohamed Ali, Mohamad Khalil, Reza Rowshan, Kamran A. Khan, Rashid K. Abu Al-Rub: Forced Convection Computational Fluid Dynamics Analysis of Architected and Three-Dimensional Printable Heat Sinks Based on Triply Periodic Minimal Surfaces

[61] Takezawa, Akihiro ; Zhang, Xiaopeng ; Kitamura, Mitsuru: Optimization of an additively manufactured functionally graded lattice structure with liquid cooling considering structural performances. In: International Journal of Heat and Mass Transfer 143 (2019), Nr. 5, S. 118564

[62] Vaissier, Benjamin ; Pernot, Jean-Philippe ; Chougrani, Laurent ; Véron, Philippe: Parametric design of graded truss lattice structures for enhanced thermal dissipation. In: Computer-Aided Design 115 (2019), Nr. 1, S. $1-12$

[63] Chantzis, Dimitrios ; Liu, Xiaochuan ; Politis, Denis J. ; Shi, Zhusheng ; Wang, Liliang: Design for additive manufacturing (DfAM) of hot stamping dies with improved cooling performance under cyclic loading conditions. In: Additive Manufacturing 37 (2021), S. 101720

[64] Kaur, Inderjot ; Singh, Prashant: Flow and Thermal Transport Through Unit Cell Topologies of Cubic and Octahedron Families. In: International Journal of Heat and Mass Transfer 158 (2020), Nr. 1, S. 119784

[65] Busse, Corinna ; Freund, Hannsjörg ; Schwieger, Wilhelm: Intensification of heat transfer in catalytic reactors by additively manufactured periodic open cellular structures (POCS). In: Chemical Engineering and Processing - Process Intensification 124 (2018), S. 199-214

[66] Wong, K. K. ; Leong, K. C.: Saturated pool boiling enhancement using porous lattice structures produced by Selective Laser Melting. In: International Journal of Heat and Mass Transfer 121 (2018), Nr. 7, S. 46-63

[67] Wong, K. K. ; Leong, K. C.: Nucleate flow boiling enhancement on engineered three-dimensional porous metallic structures in FC-72. In: Applied Thermal Engineering 159 (2019), Nr. 2, S. 113846

[68] Catchpole-Smith, S. ; Sélo, R.R.J. ; Davis, A. W. ; Ashcroft, I. A. ; Tuck, C. J. ; Clare, A.: Thermal conductivity of TPMS lattice structures manufactured via laser powder bed fusion. In: Additive Manufacturing 30 (2019), Nr. 4, S. 100846

[69] Wu, Tong ; Tovar, Andres: Multiscale, thermomechanical topology optimization of self-supporting cellular structures for porous injection molds. In: Rapid Prototyping Journal 25 (2019), Nr. 9, S. 1482-1492

[70] Essa, Khamis ; Sabouri, Aydin ; Butt, Haider ; Basuny, Fawzia Hamed ; Ghazy, Mootaz ; El-Sayed, Mahmoud Ahmed: Laser additive manufacturing of 3D meshes for optical applications. In: PloS one 13 (2018), Nr. 2, e0192389

[71] Sreedhar, Nurshaun ; Thomas, Navya ; Al-Ketan, Oraib ; Rowshan, Reza ; Hernandez, Hector H. ; Abu AlRub, Rashid K. ; Arafat, Hassan A.: Mass transfer analysis of ultrafiltration using spacers based on triply periodic minimal surfaces: Effects of spacer design, directionality and voidage. In: Journal of Membrane Science 561 (2018), S. 89-98

[72] Łosiewicz, Bożena ; Maszybrocka, Joanna ; Kubisztal, Julian ; Skrabalak, Grzegorz ; Stwora, Andrzej: Corrosion Resistance of the CpTi G2 Cellular Lattice with TPMS Architecture for Gas Diffusion Electrodes. In: Materials (Basel, Switzerland) 14 (2020), Nr. 1 